Pacific

Journal of

Mathematics

OSCILLATION AND NONOSCILLATION OF FORCED SECOND ORDER DYNAMIC EQUATIONS

MARTIN Bohner AND Christopher C. TISDELL 


\title{
OSCILLATION AND NONOSCILLATION OF FORCED SECOND ORDER DYNAMIC EQUATIONS
}

\author{
MARTin Bohner And Christopher C. TISDEll
}

\begin{abstract}
Oscillation and nonoscillation properties of second order Sturm-Liouville dynamic equations on time scales - for example, second order self-adjoint differential equations and second order Sturm-Liouville difference equations - have attracted much interest. Here we consider a given homogeneous equation and a corresponding equation with forcing term. We give new conditions implying that the latter equation inherits the oscillatory behavior of the homogeneous equation. We also give new conditions that introduce oscillation of the inhomogeneous equation while the homogeneous equation is nonoscillatory. Finally, we explain a gap in a result given in the literature for the continuous and the discrete case. A more useful result is presented, improving the theory even for the corresponding continuous and discrete cases. Examples illustrating the theoretical results are supplied.
\end{abstract}

\section{Introduction}

The theory of dynamic equations on time scales continues to be a rapidly growing area of research. Behind the main motivation for the subject lies the key concept that dynamic equations on time scales represent a way of unifying and extending continuous and discrete analysis. In this paper, we consider the second order linear dynamic equation

$$
\left(c(t) x^{\Delta}\right)^{\Delta}+q(t) x^{\sigma}=0
$$

together with an inhomogeneous equation of the form

$$
\left(c(t) u^{\Delta}\right)^{\Delta}+q(t) u^{\sigma}=f(t) .
$$

Equations (1) and (2) are so-called dynamic equations on a time scale $\mathbb{T}$. Throughout this paper we assume that $c, q$, and $f$ are rd-continuous real-valued functions defined on the time scale $\mathbb{T}$ such that $c(t) \neq 0$ for all $t \in \mathbb{T}$ and $f$ is not eventually

MSC2000: primary 34C10, 34K11, 39A11; secondary 39A10, 39A12.

Keywords: dynamic equation, generalized zero, oscillation, nonoscillation, inhomogeneous equation, time scale.

Supported by the Australian Research Council's Discovery Projects (DP0450752). 
identically equal to zero. No further assumptions on the sign of these functions are imposed. Since we are interested in oscillatory behavior of solutions of (1) and (2), we assume that the time scale $\mathbb{T}$ is unbounded above. The setup of this paper is as follows. In Section 2, we give some preliminaries concerning the time scales calculus. In Section 3, we introduce the Komkov transformation and present some basic results about equations (1) and (2). In Section 4, given that (1) is nonoscillatory, we offer criteria that introduce oscillation in (2) and also criteria that preserve nonoscillation in (2). Finally, in Section 5, we explain a gap in a result given in the continuous case by Rankin [1979, Theorem 1] and in the discrete case by Grace and El-Morshedy [1997, Theorem 2.1]. A more useful result is presented, hence improving the theory even for the corresponding continuous and discrete cases. Throughout, relevant examples illustrating the theoretical results are supplied.

\section{The time scales calculus}

In this section we present some definitions and elementary results connected to the time scales calculus. For further study we refer the reader to [Bohner and Peterson 2001; 2003]. A time scale $\mathbb{T}$ is an arbitrary nonempty closed subset of the real numbers $\mathbb{R}$. On $\mathbb{T}$ we define the forward and backward jump operators by

$$
\sigma(t):=\inf \{s \in \mathbb{T}: s>t\} \quad \text { and } \quad \rho(t):=\sup \{s \in \mathbb{T}: s<t\} \quad \text { for } \quad t \in \mathbb{T} .
$$

A point $t \in \mathbb{T}$ with $t>\inf \mathbb{T}$ is said to be left-dense if $\rho(t)=t$ and right-dense if $\sigma(t)=t$, left-scattered if $\rho(t)<t$ and right-scattered if $\sigma(t)>t$. Next, the graininess function $\mu$ is defined by $\mu(t):=\sigma(t)-t$ for $t \in \mathbb{T}$. For a function $f: \mathbb{T} \rightarrow \mathbb{R}$ the (delta) derivative $f^{\Delta}(t)$ at $t \in \mathbb{T}$ is defined to be the number (provided it exists) with the property such that for every $\varepsilon>0$ there exists a neighborhood $U$ of $t$ with

$$
\left|f(\sigma(t))-f(s)-f^{\Delta}(t)(\sigma(t)-s)\right| \leq \varepsilon|\sigma(t)-s| \quad \text { for all } \quad s \in U .
$$

A useful formula is

$$
f^{\sigma}=f+\mu f^{\Delta}, \quad \text { where } \quad f^{\sigma}:=f \circ \sigma .
$$

We will use the product rule and the quotient rule for the derivative of the product $f g$ and the quotient $f / g$ (if $g g^{\sigma} \neq 0$ ) of two differentiable functions $f$ and $g$

$$
(f g)^{\Delta}=f^{\Delta} g+f^{\sigma} g^{\Delta}=f g^{\Delta}+f^{\Delta} g^{\sigma} \quad \text { and } \quad\left(\frac{f}{g}\right)^{\Delta}=\frac{f^{\Delta} g-g^{\Delta} f}{g g^{\sigma}} .
$$

For $a, b \in \mathbb{T}$ and a function $f: \mathbb{T} \rightarrow \mathbb{R}$, the Cauchy integral of $f$ is defined by

$$
\int_{a}^{b} f(t) \Delta t=F(b)-F(a)
$$


where $F$ is an antiderivative of $f$, i.e., $F^{\Delta}=f$ holds. The function $f: \mathbb{T} \rightarrow \mathbb{R}$ is called rd-continuous if it is continuous in right-dense points and if the leftsided limits exist in left-dense points. Hilger's main existence theorem [Bohner and Peterson 2001, Theorem 1.74] says that rd-continuous functions possess antiderivatives. If $p: \mathbb{T} \rightarrow \mathbb{R}$ is rd-continuous and regressive (i.e., $1+\mu(t) p(t) \neq 0$ for all $t \in \mathbb{T}$ ), then another existence theorem says that the initial value problem $y^{\Delta}=p(t) y, y\left(t_{0}\right)=1$ (where $t_{0} \in \mathbb{T}$ ) possesses a unique solution $e_{p}\left(\cdot, t_{0}\right)$.

Example 1. Note that in the case $\mathbb{T}=\mathbb{R}$ we have

$$
\sigma(t)=t, \quad \mu(t) \equiv 0, \quad f^{\Delta}(t)=f^{\prime}(t),
$$

and in the case $\mathbb{T}=\mathbb{Z}$ we have

$$
\sigma(t)=t+1, \quad \mu(t) \equiv 1, \quad f^{\Delta}(t)=\Delta f(t)=f(t+1)-f(t) .
$$

Another important time scale is $\mathbb{T}=q^{\mathbb{N}_{0}}:=\left\{q^{k}: k \in \mathbb{N}_{0}\right\}$ with $q>1$, for which

$$
\sigma(t)=q t, \quad \mu(t)=(q-1) t, \quad f^{\Delta}(t)=\frac{f(q t)-f(t)}{(q-1) t},
$$

and this time scale gives rise to so-called $q$-difference equations.

\section{Generalized zeros and the Komkov transformation}

We say that a solution $x$ of (1) (or (2)) has a generalized zero in $[t, \sigma(t)]$ if

$$
c(t) x(t) x(\sigma(t)) \leq 0 .
$$

Next, $x$ is called oscillatory provided $[T, \infty)$ contains infinitely many zeros for each $T \in \mathbb{T}$. Otherwise we say that $x$ is nonoscillatory. The equation (1) (or (2)) is called oscillatory if all solutions of (1) (or (2)) are oscillatory. Otherwise we say that (1) (or (2)) is nonoscillatory. It is a well-known fact that (1) is oscillatory if and only if it has an oscillatory solution. The proof is easy: Suppose $x$ is a

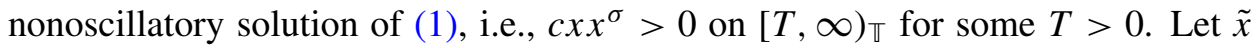
be any solution of (1) such that $x$ and $\tilde{x}$ are linearly independent. Then $(\tilde{x} / x)^{\Delta}=$ $W(x, \tilde{x}) /\left(c x x^{\sigma}\right)$ by the quotient rule (4), where $W(x, \tilde{x}):=c\left(\tilde{x}^{\Delta} x-x^{\Delta} \tilde{x}\right)$, the Wronskian, is actually equal to a nonzero constant (use the product rule (4) to verify this). Hence $\tilde{x} / x$ is eventually strictly monotone, and therefore it is eventually of one sign. Thus $\left(c \tilde{x} \tilde{x}^{\sigma}\right) /\left(c x x^{\sigma}\right)=(\tilde{x} / x)\left(\tilde{x}^{\sigma} / x^{\sigma}\right)$ is eventually positive, and hence $c \tilde{x} \tilde{x}^{\sigma}>0$ eventually, meaning that $\tilde{x}$ is nonoscillatory as well.

In contrast to (1), it is not true that (2) is oscillatory if and only if it has an oscillatory solution. We supply the following examples.

Example 2. Suppose $x$ solves (1) such that $|x(t)| \leq 1$ for all $t \in \mathbb{T}$ and such that for all $t \in \mathbb{T}$ there exist $t_{1}, t_{2} \geq t$ with $x\left(t_{1}\right)=1$ and $x\left(t_{2}\right)=-1$. Then $u_{1}:=1+x / 2$ 
is a nonoscillatory solution of (2) with $f=q$ while $u_{2}:=1+2 x$ is an oscillatory solution.

Example 3. Consider the second order linear dynamic equation

$$
\left(\mu(t) u^{\Delta}\right)^{\Delta}+\frac{4}{\mu(t)} u^{\sigma}=\frac{4}{\mu(t)}
$$

on an isolated time scale (i.e., each point is left-scattered and right-scattered). A solution of the corresponding homogeneous equation is $x=e_{-2 / \mu}\left(\cdot, t_{0}\right)$, where $t_{0} \in \mathbb{T}$. Since $x^{\sigma}=-x$, this solution is oscillatory. Hence the corresponding homogeneous equation is oscillatory. However, $u_{1}:=1+x / 2$ is a nonoscillatory solution of (6) while $u_{2}:=1+2 x$ is an oscillatory solution of (6). Hence (6) possesses both oscillatory and nonoscillatory solutions.

The transformation $u=x y$, where $x$ solves (1) and $u$ solves (2), was studied by Komkov [1972] and has been successfully applied, for example, in [Grace and El-Morshedy 1997; Patula 1979; Rankin 1979]. Our results given in this paper mainly rely on the following easy but useful identity. We abbreviate the operator $\left(c x^{\Delta}\right)^{\Delta}+q x^{\sigma}$ by $L x$.

Lemma 1. If $u=x y$, then

$$
W(x, u)=c x x^{\sigma} y^{\Delta}
$$

and

$$
[W(x, u)]^{\Delta}=x^{\sigma} L u-u^{\sigma} L x .
$$

In particular, if $x$ solves (1) and $u$ solves (2), then

$$
[W(x, u)]^{\Delta}=\left(c x x^{\sigma} y^{\Delta}\right)^{\Delta}=f x^{\sigma},
$$

and if in addition $x(t) \neq 0$ for all $t \geq T$, then

(9) $y(t)=y(T)+c(T) x(T) x(\sigma(T)) y^{\Delta}(T) \int_{T}^{t} \frac{\Delta s}{c(s) x(s) x(\sigma(s))}$

$$
+\int_{T}^{t} \frac{1}{c(s) x(s) x(\sigma(s))} \int_{T}^{s} f(\tau) x(\sigma(\tau)) \Delta \tau \Delta s \quad \text { for all } t \geq T .
$$

Proof. We apply the product rule (4) to $u=x y$ to find $u^{\Delta}=x^{\Delta} y+x^{\sigma} y^{\Delta}$ and

$$
c x x^{\sigma} y^{\Delta}=c x u^{\Delta}-c x x^{\Delta} y=c u^{\Delta} x-c x^{\Delta} u=W(x, u) .
$$

Then, using the product rule again, we find

$$
\begin{aligned}
\left(c x x^{\sigma} y^{\Delta}\right)^{\Delta} & =\left(c u^{\Delta}\right)^{\Delta} x^{\sigma}+c u^{\Delta} x^{\Delta}-\left(c x^{\Delta}\right)^{\Delta} u^{\sigma}-c x^{\Delta} u^{\Delta} \\
& =\left(L u-q u^{\sigma}\right) x^{\sigma}-\left(L x-q x^{\sigma}\right) u^{\sigma} \\
& =x^{\sigma} L u-u^{\sigma} L x .
\end{aligned}
$$


Hence, if $L x=0$ and $L u=f$, (8) follows. By using the definition (5) of the integral, we conclude that (9) holds.

Concluding this section, we use Lemma 1 to derive the following result. For the continuous version see [Rankin 1979, Equation $\left(3^{\prime}\right)$ ] and for the discrete version see [Patula 1979, Theorem 6] and [Grace and El-Morshedy 1997, Lemma 2.1].

Theorem 1. Suppose $x$ solves (1) and $u$ solves (2). If $W(x, u)$ is eventually of one sign (either positive or negative), then $x$ oscillates if and only if $u$ oscillates.

Proof. Using (7), we know that $W(x, u)=c x x^{\sigma} y^{\Delta}$ is eventually of one sign, where $u=x y$. First suppose $x$ is not oscillatory, i.e., $c x x^{\sigma}>0$ eventually. Hence $y^{\Delta}$ is eventually of one sign. Therefore $y$ is eventually of one sign. Hence

$$
0<y y^{\sigma}=\frac{u}{x} \frac{u^{\sigma}}{x^{\sigma}}=\frac{c u u^{\sigma}}{c x x^{\sigma}} \quad \text { eventually, }
$$

so eventually $\mathrm{cuu}^{\sigma}>0$, i.e., $u$ is not oscillatory. Similarly (by considering the transformation $x=u \tilde{y}$ ) we may show that if $u$ is not oscillatory, then $x$ is not oscillatory either.

Corollary 1. Suppose $c(t)>0$ for all $t \in \mathbb{T}$. If $(1)$ is nonoscillatory and $f$ is eventually of one sign, then (2) is nonoscillatory.

Proof. Let $x$ be any (nonoscillatory) solution of (1) so $x$ is eventually of one sign. Suppose $u$ is any solution of (2) and let $y=u / x$. By (8), $[W(x, u)]^{\Delta}$ is eventually of one sign, and hence $W(x, u)$ is eventually of one sign. Thus $u$ is nonoscillatory according to Theorem 1 .

Corollary 2. Suppose (1) is oscillatory (nonoscillatory). If there exists a solution $x$ of (1) such that

$$
\int_{T}^{\infty} f(t) x(\sigma(t)) \Delta t=\infty \quad \text { or } \quad \int_{T}^{\infty} f(t) x(\sigma(t)) \Delta t=-\infty
$$

then (2) is oscillatory (nonoscillatory).

Proof. Suppose $u$ is a solution of (2) and define $y$ by $u=x y$. By (8),

$$
W(x, u)(t)=W(x, u)(T)+\int_{T}^{t} f(s) x(\sigma(s)) \Delta s .
$$

Hence $W(x, u)$ is eventually of one sign, and the claim follows with Theorem 1 .

Example 4. Consider the Fibonacci difference equation $x(t+2)=x(t+1)+x(t), \quad$ i.e., $\quad \Delta\left((-1)^{t+1} \Delta x(t)\right)+(-1)^{t+1} x(t+1)=0, \quad t \in \mathbb{N}$. If $a=(1+\sqrt{5}) / 2$, then $x(t)=a^{t}$ is a solution of this equation. Since

$$
c(t) x(t) x(t+1)=(-a)\left(-a^{2}\right)^{t},
$$


the equation is oscillatory. Now, since $\sum_{\tau=0}^{\infty} a^{\tau+1}=\infty$, Corollary 2 implies that $x(t+2)=x(t+1)+x(t)+(-1)^{t}$, i.e., $\Delta\left((-1)^{t+1} \Delta x(t)\right)+(-1)^{t+1} x(t+1)=1$, is also oscillatory.

\section{Oscillation and nonoscillation criteria}

The next theorem generalizes a result due to Rankin for $\mathbb{T}=\mathbb{R}[$ Rankin 1979, Theorem 2] and a result due to Grace and El-Morshedy for $\mathbb{T}=\mathbb{Z}$ [Grace and El-Morshedy 1997, Theorem 2.2].

Theorem 2. Suppose $x$ is an eventually nonoscillatory solution of (1). If for some sufficiently large $T \in \mathbb{T}$,

$$
\begin{gathered}
\int_{T}^{\infty} \frac{\Delta t}{c(t) x(t) x(\sigma(t))}<\infty, \\
\liminf _{t \rightarrow \infty} \int_{T}^{t} \frac{1}{c(s) x(s) x(\sigma(s))} \int_{T}^{s} f(\tau) x(\sigma(\tau)) \Delta \tau \Delta s=-\infty,
\end{gathered}
$$

and

$$
\limsup _{t \rightarrow \infty} \int_{T}^{t} \frac{1}{c(s) x(s) x(\sigma(s))} \int_{T}^{s} f(\tau) x(\sigma(\tau)) \Delta \tau \Delta s=\infty,
$$

then (2) is oscillatory.

Proof. Suppose $u$ is an eventually nonoscillating solution of (2) such that $y=u / x$ is eventually of one sign (note that $y y^{\sigma}=\left(c u u^{\sigma}\right) /\left(c x x^{\sigma}\right)>0$ ). But (9) together with (10), (11), and (12) ensures that

$$
\liminf _{t \rightarrow \infty} y(t)=-\infty \quad \text { and } \quad \limsup _{t \rightarrow \infty} y(t)=\infty .
$$

This is a contradiction, and therefore there cannot exist an eventually nonoscillating solution of (2). Thus (2) is oscillatory.

Example 5. Let $q>1$ and consider the $q$-difference equation (see Example 1)

$$
u^{\Delta \Delta}=(-1)^{\log _{q} t}, \quad t \in q^{\mathbb{N}_{0}}:=\left\{q^{k}: k \in \mathbb{N}_{0}\right\} .
$$

One solution of the corresponding homogeneous equations is $x(t)=t$, so the homogeneous equation is nonoscillatory and (10) is satisfied since

$$
\int_{1}^{t} \frac{\Delta s}{c(s) x(s) x(\sigma(s))}=1-\frac{1}{t} \rightarrow 1 \quad \text { as } \quad t \rightarrow \infty .
$$

Some calculation now shows that

$$
\int^{t} \frac{1}{q s^{2}} \int^{s} q \tau(-1)^{\log _{q} \tau} \Delta \tau \Delta s=\frac{(q-1)^{2}}{q^{3}+q^{2}+q+1} t(-1)^{\log _{q} t},
$$


so (11) and (12) are satisfied. Hence, by Theorem 2, (13) is oscillatory.

Now we present an improvement of Theorem 1. For $\mathbb{T}=\mathbb{Z}$, see [Grace and El-Morshedy 1997, Theorem 2.3].

Theorem 3. Suppose $x$ solves (1) such that $x(t) \neq 0$ for all $t \geq T$ and

$$
\int_{T}^{t} \frac{\Delta s}{c(s) x(s) x(\sigma(s))} \quad \text { is bounded above or below. }
$$

If

$$
\int_{T}^{\infty} \frac{1}{c(t) x(t) x(\sigma(t))} \int_{T}^{t} f(s) x(\sigma(s)) \Delta s \Delta t=\infty,
$$

then (1) and (2) either are both oscillatory or both nonoscillatory.

Proof. Let $u$ be a solution of (2). By Theorem 1, we may assume that $W(x, u)$ is oscillating. First suppose that the integral in (14) is bounded below. Let $T \in \mathbb{T}$ and $D>0$ be such that

$$
W(x, u)(T) \geq 0 \quad \text { and } \quad \int_{T}^{t} \frac{\Delta s}{c(s) x(s) x(\sigma(s))} \geq-D \text { for all } t \geq T .
$$

Then, by (9),

$$
y(t) \geq y(T)-D W(x, u)(T)+\int_{T}^{t} \frac{1}{c(s) x(s) x(\sigma(s))} \int_{T}^{s} f(\tau) x(\sigma(\tau)) \Delta \tau \Delta s,
$$

so $y(t) \rightarrow \infty$ as $t \rightarrow \infty$ by (15). Hence $y>0$ eventually and $c u u^{\sigma}$ has eventually the same sign as $c x x^{\sigma}$. If, however, the integral in (14) is bounded above, then we pick $T \in \mathbb{T}$ and $E>0$ such that

$$
W(x, u)(T) \leq 0 \quad \text { and } \quad \int_{T}^{t} \frac{\Delta s}{c(s) x(s) x(\sigma(s))} \leq E \quad \text { for all } \quad t \geq T .
$$

In this case the conclusion now follows as in the previous case.

The last result in this section is a nonoscillation criterion. We refer to [Grace and El-Morshedy 1997, Theorem 3.1] for $\mathbb{T}=\mathbb{Z}$. The following auxiliary result is needed.

Lemma 2. Suppose (1) is nonoscillatory. Then there exists a solution $x$ of (1) satisfying (10).

Proof. Let $x$ be any (nonoscillatory) solution of (1). If $x$ satisfies (10), then we are done. If not, then $\int_{T}^{\infty} \Delta s /(c(s) x(s) x(\sigma(s)))=\infty$. Let $\tilde{x}$ be any solution of (1) such that $x$ and $\tilde{x}$ are linearly independent, i.e., $W(x, \tilde{x}) \equiv-k \neq 0$. Then 
$(\tilde{x} / x)^{\Delta}=k /\left(c x x^{\sigma}\right)$, so $(\tilde{x} / x)(t) \rightarrow \pm \infty$ as $t \rightarrow \infty$ and hence $(x / \tilde{x})(t) \rightarrow 0$ as $t \rightarrow \infty$. Thus $(x / \tilde{x})^{\Delta}=-k /\left(c \tilde{x} \tilde{x}^{\sigma}\right)$ and therefore

$$
k \int_{T}^{t} \frac{\Delta s}{c(s) \tilde{x}(s) \tilde{x}(\sigma(s))}=\frac{x(T)}{\tilde{x}(T)}-\frac{x(t)}{\tilde{x}(t)} \rightarrow \frac{x(T)}{\tilde{x}(T)} \quad \text { as } \quad t \rightarrow \infty .
$$

Hence $\tilde{x}$ solves (1) and satisfies (10).

Below we use for $\alpha \in \mathbb{R}$ the notation $\alpha^{+}=\max \{0, \alpha\}$ and $\alpha^{-}=\min \{0, \alpha\}$.

Theorem 4. Suppose $c(t)>0$ and $q(t)>0$ for all $t \in \mathbb{T}$. Suppose that all solutions of (1) are nonoscillatory and bounded. If

$$
\int_{T}^{\infty} \frac{1}{c(s)} \int_{T}^{s} f^{+}(\tau) \Delta \tau \Delta s=\infty
$$

and

$$
\int_{T}^{\infty} f^{-}(\tau) \Delta \tau>-\infty
$$

then (2) is nonoscillatory.

Proof. Let $\lambda>0$ be such that the integral in (17) is bounded below by $-\lambda$. By Lemma 2, there exists a solution $x$ of (1) satisfying (10). Since all solutions of (1) are bounded, there exists $M>0$ such that $|x(t)|<M$ for all $t \in \mathbb{T}$. We will show that (15) is satisfied. Then Theorem 3 is employed to complete the proof.

First, putting $z=c x^{\Delta}$, we see that $z^{\Delta}=-q x^{\sigma}$ is eventually of one sign. Thus $z$ is eventually of one sign. Hence $x^{\Delta}$ is eventually of one sign. Therefore $x$ is eventually increasing or eventually decreasing. Since $x$ is nonoscillatory, it is either eventually positive or eventually negative. So there are the following four possibilities: (i) $x$ is eventually positive and increasing; (ii) $x$ is eventually positive and decreasing; (iii) $x$ is eventually negative and increasing; (iv) $x$ is eventually negative and decreasing. If (iii) or (iv) holds, then we may replace $x$ by $-x$, which is also a solution of (1) that satisfies (10) and (i) or (ii). Thus it is sufficient to discuss the cases (i) and (ii). If (i) holds, then

$$
\begin{aligned}
\int_{T}^{t} \frac{1}{c(s) x(s) x(\sigma(s))} \int_{T}^{s} f(\tau) x(\sigma(\tau)) \Delta \tau \Delta s & \\
\geq \frac{x(\sigma(T))}{M^{2}} \int_{T}^{t} \frac{1}{c(s)} \int_{T}^{s} f^{+}(\tau) \Delta \tau \Delta s-\lambda M \int_{T}^{t} \frac{\Delta s}{c(s) x(s) x(\sigma(s))} & \stackrel{(16)}{\rightarrow} \infty \text { as } t \rightarrow \infty
\end{aligned}
$$


(use $f=f^{+}+f^{-}$), while if (ii) holds, then

$$
\begin{aligned}
& \int_{T}^{t} \frac{1}{c(s) x(s) x(\sigma(s))} \int_{T}^{s} f(\tau) x(\sigma(\tau)) \Delta \tau \Delta s \\
& \geq \int_{T}^{t} \frac{1}{c(s) x(s) x(\sigma(s))} \int_{T}^{s} f^{+}(\tau) x(\sigma(s)) \Delta \tau \Delta s-\lambda M \int_{T}^{t} \frac{\Delta s}{c(s) x(s) x(\sigma(s))} \\
& \geq \frac{1}{M} \int_{T}^{t} \frac{1}{c(s)} \int_{T}^{s} f^{+}(\tau) \Delta \tau \Delta s-\lambda M \int_{T}^{t} \frac{\Delta s}{c(s) x(s) x(\sigma(s))} \\
& \stackrel{(16)}{\rightarrow} \infty \text { as } t \rightarrow \infty .
\end{aligned}
$$

Hence (15) holds in either case and the proof is complete.

\section{Remarks on the results of Rankin, Grace and El-Morshedy}

The continuous version of the following result was proved by Rankin [1979, Theorem 1], while its discrete version was given by Grace and El-Morshedy in [1997, Theorem 2.1].

Theorem 5. Suppose $x$ is an eventually nonoscillatory solution of (1). If for sufficiently large $T \in \mathbb{T}$ and some $M>0$,

$$
\begin{gathered}
\int_{T}^{\infty} \frac{\Delta t}{c(t) x(t) x(\sigma(t))}=\infty \\
\liminf _{t \rightarrow \infty} \int_{T}^{t} f(s) x(\sigma(s)) \Delta s=-\infty, \quad \limsup _{t \rightarrow \infty} \int_{T}^{t} f(s) x(\sigma(s)) \Delta s=\infty,
\end{gathered}
$$

and

$$
\left|\int_{T}^{t} \frac{1}{c(s) x(s) x(\sigma(s))} \int_{T}^{s} f(\tau) x(\sigma(\tau)) \Delta \tau \Delta s\right| \leq M \int_{T}^{t} \frac{\Delta s}{c(s) x(s) x(\sigma(s))}
$$

for all $t \geq T$, then (2) is oscillatory.

Below we show that the assumptions of Theorem 5 are never satisfied. This means that, although Theorem 5 is true, the result is not meaningful.

Theorem 6. The assumptions of Theorem 5 are never satisfied.

Proof. Note first that (2) has a solution. Let $u$ be any solution of (2) and define $y$ by $u=x y$. Let $T \in \mathbb{T}$ such that $c(t) x(t) x(\sigma(t)) \geq 0$ for all $t \geq T$. By (8), we have

$$
c(t) x(t) x(\sigma(t)) y^{\Delta}(t)=c(T) x(T) x(\sigma(T)) y^{\Delta}(T)+\int_{T}^{t} f(\tau) x(\sigma(\tau)) \Delta \tau,
$$

so (19) implies

(21) $\liminf _{t \rightarrow \infty} c(t) x(t) x(\sigma(t)) y^{\Delta}(t)=-\infty, \quad \limsup _{t \rightarrow \infty} c(t) x(t) x(\sigma(t)) y^{\Delta}(t)=\infty$. 
By the first relation in (21), there exists $\tilde{T} \geq T$ with $c(\tilde{T}) x(\tilde{T}) x(\sigma(\tilde{T})) y^{\Delta}(\tilde{T})<$ $-2 M$. Thus, using (8), we find

$$
\begin{aligned}
c(t) x(t) x(\sigma(t)) y^{\Delta}(t) & =c(\tilde{T}) x(\tilde{T}) x(\sigma(\tilde{T})) y^{\Delta}(\tilde{T})+\int_{\tilde{T}}^{t} f(\tau) x(\sigma(\tau)) \Delta \tau \\
& <-2 M+\int_{\tilde{T}}^{t} f(\tau) x(\sigma(\tau)) \Delta \tau
\end{aligned}
$$

and hence

$y(t)$

$$
\begin{aligned}
& <y(\tilde{T})-2 M \int_{\tilde{T}}^{t} \frac{\Delta s}{c(s) x(s) x(\sigma(s))}+\int_{\tilde{T}}^{t} \frac{1}{c(s) x(s) x(\sigma(s))} \int_{\tilde{T}}^{s} f(\tau) x(\sigma(\tau)) \Delta \tau \Delta s \\
& \stackrel{(20)}{\leq} y(\tilde{T})-M \int_{\tilde{T}}^{t} \frac{\Delta s}{c(s) x(s) x(\sigma(s))} \stackrel{(18)}{\rightarrow}-\infty \quad \text { as } \quad t \rightarrow \infty .
\end{aligned}
$$

By the second relation in (21), there exists $\bar{T} \geq T$ with $c(\bar{T}) x(\bar{T}) x(\sigma(\bar{T})) y^{\Delta}(\bar{T})>$ $2 M$. Thus, using (8), we find

$$
\begin{aligned}
c(t) x(t) x(\sigma(t)) y^{\Delta}(t) & =c(\bar{T}) x(\bar{T}) x(\sigma(\bar{T})) y^{\Delta}(\bar{T})+\int_{\bar{T}}^{t} f(\tau) x(\sigma(\tau)) \Delta \tau \\
& >2 M+\int_{\bar{T}}^{t} f(\tau) x(\sigma(\tau)) \Delta \tau
\end{aligned}
$$

and hence

$y(t)$

$$
\begin{aligned}
& >y(\bar{T})+2 M \int_{\bar{T}}^{t} \frac{\Delta s}{c(s) x(s) x(\sigma(s))}+\int_{\bar{T}}^{t} \frac{1}{c(s) x(s) x(\sigma(s))} \int_{\bar{T}}^{s} f(\tau) x(\sigma(\tau)) \Delta \tau \Delta s \\
& \stackrel{(20)}{\geq} y(\bar{T})+M \int_{\bar{T}}^{t} \frac{\Delta s}{c(s) x(s) x(\sigma(s))} \stackrel{(18)}{\rightarrow} \infty \quad \text { as } \quad t \rightarrow \infty .
\end{aligned}
$$

This is a contradiction, as $y(t) \rightarrow \infty$ and $y(t) \rightarrow-\infty$ at the same time for $t \rightarrow \infty$.

Example 6. Rankin [1979, Example 2] stated that Theorem 5 for the case $\mathbb{T}=\mathbb{R}$ can be used to show that

$$
u^{\prime \prime}=t \sin t, \quad t \in \mathbb{R}
$$

is oscillatory. Here we let $x(t) \equiv 1$. Clearly, conditions (18) and (19) are satisfied. A simple calculation shows that

$$
\left|\int_{T}^{t} \int_{T}^{s} \tau \sin \tau \mathrm{d} \tau \mathrm{d} s\right| \leq(2 T+4)(t-T),
$$


so (20) is satisfied if $M$ is allowed to depend on $T$. However, if $M=M(T)$, then the proof of Theorem 5 (and Theorem 6) breaks down. Furthermore, the equation (22) is in fact not oscillatory: Clearly,

$$
u_{1}(t)=4-2 \cos (t)+t(2-\sin t) \text { and } u_{2}(t)=-t \sin t-2 \cos t
$$

both are solutions of (22), and $u_{1}$ is nonoscillatory while $u_{2}$ is oscillatory.

Example 7. We note that Grace and El-Morshedy [1997] did not supply an example to illustrate Theorem 5 for the case $\mathbb{T}=\mathbb{Z}$. Consider the difference equation

$$
\Delta^{2} u=(-1)^{t+1}(2 t+1), \quad t \in \mathbb{N} .
$$

Here we let $x(t) \equiv 1$. Clearly, conditions (18) and (19) are satisfied. A simple calculation shows that

$$
\left|\sum_{s=T}^{t-1} \sum_{\tau=T}^{s-1}(-1)^{\tau+1}(2 \tau+1)\right| \leq 2 T(t-T)
$$

for $T \in \mathbb{N}$, so (20) is satisfied if $M$ is allowed to depend on $T$. Furthermore, the equation (22) is in fact not oscillatory: Clearly,

$$
u_{1}(t)=t+(-1)^{t+1}\left[\frac{t}{2}\right] \quad \text { and } \quad u_{2}(t)=(-1)^{t+1}\left[\frac{t}{2}\right],
$$

where $[x]$ denotes the largest integer less than or equal to $x \in \mathbb{R}$, both are solutions of (22), and $u_{1}$ is nonoscillatory while $u_{2}$ is oscillatory.

We now present the following results.

Theorem 7. Let $T \in \mathbb{T}$. Assume $x$ is any solution of $(1)$ with $c(t) x(t) x(\sigma(t))>0$ for all $t \geq T$. If (18) holds and if there exists $M>0$ such that (20) is satisfied, then (2) is not oscillatory.

Proof. Define

$$
y(t):=2 M \int_{T}^{t} \frac{\Delta s}{c(s) x(s) x(\sigma(s))}+\int_{T}^{t} \frac{1}{c(s) x(s) x(\sigma(s))} \int_{T}^{s} f(\tau) x(\sigma(\tau)) \Delta \tau \Delta s .
$$

Using the product and the quotient rule (4), it is easy to check that $u$ defined by $u:=y x$ is a solution of (2). However, (20) ensures that $\lim _{t \rightarrow \infty} y(t)=\infty$, and therefore $u$ is a nonoscillatory solution of (2). Thus (2) cannot be oscillatory.

Theorem 8. Let $T \in \mathbb{T}$. Assume $x$ is any solution of $(1)$ with $c(t) x(t) x(\sigma(t))>0$ for all $t \geq T$. If (18) holds, if there exists $M>0$ such that (20) is satisfied, and if (11) and (12) hold, then (2) has both oscillatory and nonoscillatory solutions. 
Proof. Define

$$
y_{1}(t):=2 M \int_{T}^{t} \frac{\Delta s}{c(s) x(s) x(\sigma(s))}+\int_{T}^{t} \frac{1}{c(s) x(s) x(\sigma(s))} \int_{T}^{s} f(\tau) x(\sigma(\tau)) \Delta \tau \Delta s
$$

and

$$
y_{2}(t):=\int_{T}^{t} \frac{1}{c(s) x(s) x(\sigma(s))} \int_{T}^{s} f(\tau) x(\sigma(\tau)) \Delta \tau \Delta s .
$$

As in the proof of Theorem 7, it is easy to check that $u_{1}$ and $u_{2}$ defined by $u_{1}:=y_{1} x$ and $u_{2}:=y_{2} x$ both are solutions of (2). While $u_{1}$ is nonoscillatory, $u_{2}$ is oscillatory. Hence (2) indeed has both oscillatory and nonoscillatory solutions.

Our next result can be checked easily as in the proof of Theorem 7 .

Theorem 9. Suppose that the solution $x$ of (1) satisfies $x(t) \neq 0$ for all $t \geq T$. Then the solution of (2) satisfying the initial conditions $u(T)=\alpha$ and $u^{\Delta}(T)=\beta$ is given by $u:=y x$, where

$y(t)=\gamma+\delta \int_{T}^{t} \frac{\Delta s}{c(s) x(s) x(\sigma(s))}+\int_{T}^{t} \frac{1}{c(s) x(s) x(\sigma(s))} \int_{T}^{s} f(\tau) x(\sigma(\tau)) \Delta \tau \Delta s$, where $\gamma=\alpha / x(T)$ and $\delta=c(T)\left(\beta x(T)-\alpha x^{\Delta}(T)\right)$.

The following generalization of Theorem 2 now becomes apparent.

Theorem 10. Suppose $x$ is a nonoscillatory solution of (1). If for some $T \in \mathbb{T}$,

$$
\liminf _{t \rightarrow \infty} \frac{\int_{T}^{t} \frac{1}{c(s) x(s) x(\sigma(s))} \int_{T}^{s} f(\tau) x(\sigma(\tau)) \Delta \tau \Delta s}{\int_{T}^{t} \frac{\Delta s}{c(s) x(s) x(\sigma(s))}}=-\infty
$$

and

$$
\limsup _{t \rightarrow \infty} \frac{\int_{T}^{t} \frac{1}{c(s) x(s) x(\sigma(s))} \int_{T}^{s} f(\tau) x(\sigma(\tau)) \Delta \tau \Delta s}{\int_{T}^{t} \frac{\Delta s}{c(s) x(s) x(\sigma(s))}}=\infty,
$$

then (2) is oscillatory.

\section{References}

[Bohner and Peterson 2003] M. Bohner and A. Peterson (editors), Advances in dynamic equations on time scales, Birkhäuser, Boston, 2003. MR 2004d:34003 Zbl 1025.34001

[Bohner and Peterson 2001] M. Bohner and A. Peterson, Dynamic equations on time scales: An introduction with applications, Birkhäuser, Boston, 2001. MR 2002c:34002 Zbl 0978.39001

[Grace and El-Morshedy 1997] S. R. Grace and H. A. El-Morshedy, "Oscillation and nonoscillation theorems for certain second-order difference equations with forcing term", J. Math. Anal. Appl. 216:2 (1997), 614-625. MR 99d:39005 Zbl 0896.39004 
[Komkov 1972] V. Komkov, "A technique for the detection of oscillation of second order ordinary differential equations", Pacific J. Math. 42 (1972), 105-115. MR 47 \#2134 Zbl 0241.34038

[Patula 1979] W. T. Patula, "Growth, oscillation and comparison theorems for second-order linear difference equations”, SIAM J. Math. Anal. 10 (1979), 1272-1279. MR 80j:39004 Zbl 0433.39005

[Rankin 1979] S. M. Rankin, "Oscillation results for a nonhomogeneous equation", Pacific J. Math. 80:1 (1979), 237-243. MR 80h:34044 Zbl 0407.34029

Received June 7, 2005.

MARTIN BOHNER

DEPARTMENT OF MATHEMATICS

UNIVERSITY OF MISSOURI

ROLLA, MO 65401

UNITED STATES

bohner@umr.edu

Christopher C. TISDEll

SCHOOL OF MATHEMATICS AND STATISTICS

THE UNIVERSity OF NeW SOUTh WALES

SYDNEY, NSW 2052

Australia

cct@maths.unsw.edu.au 